\title{
THE HILL-PENROSE-SPARLING CR MANIFOLDS
}

\author{
MICHAEL G. EASTWOOD
}

(Communicated by Irwin Kra)

\begin{abstract}
This paper gives a simple formulation of the CR structures of Hill, Penrose, and Sparling. An elementary power series argument shows that they cannot be realized as hypersurfaces in an ambient complex manifold.
\end{abstract}

Suppose $M$ is a smooth three-dimensional real manifold equipped with a nowhere vanishing complex vector field $X$ and a smooth complex-valued function $g$ such that, even locally, the equation $X f=g$ has no solutions. One may regard $X$ as defining a CR structure on $M$ and then $g$ represents a nowhere vanishing class in the $\bar{\partial}_{b}$ cohomology $H^{0,1}(M)$. As described in [2], this class may be exponentiated to a CR line bundle over $M$ with total space $T$. This example $T$ of a CR manifold is due to Hill, Penrose, and Sparling who have shown (see [2]) that it cannot be locally realized as a hypersurface in $\mathbf{C}^{3}$. (An alternative proof of this fact is given by Jacobowitz [1].)

The manifold $T$ may be defined as $M \times \mathbf{C}$ with $\mathrm{CR}$ structure induced by the vector fields

$$
X+g z \partial / \partial z \text { and } \partial / \partial \bar{z}
$$

where $z$ is the usual coordinate on $\mathbf{C}$. To show that this is nonrealizable, first notice that if a smooth function $\alpha$ satisfies $X \alpha=n g \alpha$, where $n$ is a nonzero constant, then $\alpha$ must be identically zero for, otherwise, any local choice of $n^{-1} \log \alpha$ would provide a solution of $X f=g$. Suppose that $h$ is CR holomorphic on $T$. In other words,

$$
X h+g z \partial h / \partial z=0=\partial h / \partial \bar{z} .
$$

The second equation says that this function is holomorphic in the variable $z$ and so may be expanded as a Taylor series $h=\sum h_{j}(x) z^{j}$ with coefficients $h_{j}$ depending smoothly on $x \in M$. One may apply $X$ term by term and the second equation yields

$$
X h_{j}+j g h_{j}=0 \text { for all } j .
$$

As noticed above, the forces $h_{j} \equiv 0$ for $j \geq 1$. Thus, $h$ is independent of $z: h=h(x)$ for $h(x)$ a CR function on $M$. Hence, the CR functions fail to separate points as they would do if $T$ were a hypersurface in $\mathbf{C}^{3}$.

As noted by Jacobowitz [1] the CR manifold $M$ need not be restricted to have dimension three. The only requirement is that $H^{0,1}(M)$ be nonzero and the above argument easily generalizes to cover this case. For example, $M$ can be taken to be a hypersurface of Levi type $(1, k)$. The manifold $T=M \times \mathbf{C}$ is always Levi flat in the $\mathbf{C}$ direction.

Received by the editors July 3, 1986 and, in revised form, December 9, 1986.

1980 Mathematics Subject Classification (1985 Revision). Primary 32C10; Secondary 58A30, $58 \mathrm{G} 30$. 


\section{REFERENCES}

1. H. Jacobowitz, Simple examples of non-realizable CR hypersurfaces, Preprint.

2. R. Penrose, Physical space-time and non-realizable CR-structures, Bull. Amer. Math. Soc. (N.S.) 8 (1983), 427-448.

Department of PURE MAThematics, UNiversity of Adelaide, G.P.O. Box 498, Adelaide, South Australia 5001, Australia 